\title{
Role of Conjunctival Ultraviolet Autofluorescence in Ocular Surface Squamous Neoplasia
}

\author{
Saumya Yadav ${ }^{a}$ Noopur Gupta ${ }^{a}$ Rashmi Singh ${ }^{a}$ Mukesh Patil ${ }^{a}$ \\ Rachna Meel $^{b}$ Murugesan Vanathi ${ }^{a}$ Seema Kashyap ${ }^{c}$ Radhika Tandon $^{\text {a }}$ \\ ${ }^{a}$ Cataract, Cornea, Refractive and Ocular Oncology Services, Dr. Rajendra Prasad Centre for Ophthalmic Sciences, \\ All India Institute of Medical Sciences, New Delhi, India; b Oculoplasty and Ocular Oncology Services, \\ Dr. Rajendra Prasad Centre for Ophthalmic Sciences, All India Institute of Medical Sciences, New Delhi, India; \\ 'Ocular Pathology Services, Dr. Rajendra Prasad Centre for Ophthalmic Sciences, All India Institute of Medical \\ Sciences, New Delhi, India
}

\section{Keywords}

Autofluorescence $\cdot$ Ocular surface squamous

neoplasia · Novel biomarker · Non-invasive detection ·

Resource-friendly tool

\begin{abstract}
Objective: To evaluate the adjunctive role of conjunctival autofluorescence in the management of ocular surface squamous neoplasia (OSSN). Materials and Methods: Seventeen patients with clinically diagnosed OSSN were included. Morphological characteristics, type of OSSN, and autofluorescence photographs of the lesion were captured. Presence and area of conjunctival ultraviolet autofluorescence (CUVAF) were the main outcome measures. Results: Overall, 17 patients with $15(88 \%)$ primary and $2(12 \%)$ recurrent OSSN were included. Common locations were temporal $(n=10)$, nasal $(n=5)$, and diffuse variety $(n=2)$. Morphologically, there were 4 (22.2\%) nodular, 4 (22.2\%) leucoplakic, 3 (16.7\%) gelatinous, and 1 (5.5\%) each of papillary, nodulo-ulcerative, and diffuse variety. Mixed morphology was present in 4 eyes
\end{abstract}

(22.2\%). Sixteen of 18 eyes (88.9\%) with OSSN displayed autofluorescence on CUVAF images. The mean area of CUVAF was $15.82 \mathrm{~mm}^{2}\left(10.77-19.59 \mathrm{~mm}^{2}\right)$. Autofluorescence was reported in 8 eyes (44.4\%) which had negative reports on impression cytology. Conclusions: Conjunctival autofluorescence was seen in the majority of cases with OSSN, in spite of negative cytology reports. Our study demonstrates that CUVAF may serve as an effective ancillary, non-invasive, and resource-friendly tool for supplementing the clinical diagnosis of OSSN, especially in diffuse and recurrent lesions that are not amenable to surgical intervention.

(c) 2020 S. Karger AG, Basel

\section{Introduction}

Ocular surface squamous neoplasia (OSSN) is the most common malignant tumour [1] of the conjunctival epithelium with a potential to cause extensive ocular damage and permanent visual loss. Its spectrum ranges from conjunctival intraepithelial neoplasia characterized karger@karger.com

(c) 2020 S. Karger AG, Basel

Karger! 
by dysplastic changes of the ocular surface epithelium to invasive squamous cell carcinoma invading the substantia propria of the conjunctiva or Bowman's layer of the cornea [2]. Early diagnosis and timely management play a crucial role in improving the prognosis and outcome in patients with OSSN. The aetiology of OSSN is multifactorial with risk factors including ultraviolet radiation exposure [3, 4], xeroderma pigmentosa [5], and infections with human immunodeficiency virus and human papilloma virus [6-8]. In tropical countries such as India, OSSN mainly affects older males with a history of prolonged sun exposure. Ultraviolet radiation leads to solar elastosis, a risk factor for dysplastic and neoplastic changes in the corneal and conjunctival epithelium $[3,4,9]$.

Conjunctival ultraviolet autofluorescence (CUVAF) imaging has been developed as an objective biomarker of ocular ultraviolet radiation exposure. It quantifies the autofluorescent area that is presumably emitted by endogenous cellular components when they are excited by ultraviolet radiation of appropriate wavelength [10]. Its role has been described in ascertaining time spent outdoors [11], early pterygia [12], pinguecula [13], myopia [14], and ocular sun exposure [15]. To the best of our knowledge, the relationship between CUVAF and OSSN in vivo has never been studied. We, therefore, undertook the present study to report the presence of autofluorescence, if any, and the spectrum of presentation patterns in different morphological variants of OSSN.

\section{Materials and Methods}

This was a cross-sectional, observational study conducted at the Dr. Rajendra Prasad Centre for Ophthalmic Sciences, All India Institute of Medical Sciences, New Delhi, India.

Patients with a clinical diagnosis of OSSN were recruited from the outpatient and ocular oncology services of our institution. These included both treatment-naïve and recurrent OSSN. The study procedures were explained to each participant in detail and a written informed consent was obtained from all the patients. Histopathological confirmation of all cases that underwent surgical excision was performed. In cases with diffuse lesions not amenable to surgical intervention, positive response to therapy was noted and the diagnosis confirmed to exclude conditions like pterygium, squamous metaplasia, reactive hyperplasia, scars, calcifications, and inclusion cysts, that may mimic cases of early OSSN. Cases of OSSN already on chemotherapy/immunotherapy, those with intraocular/orbital involvement, and those in whom the posterior extent of the lesion could not be defined were excluded from the study.

Following recruitment, the demographic and clinical details including associated systemic illness, previous treatment history, and best corrected visual acuity of the patient were noted at the time of presentation. Detailed anterior segment examination on slit lamp biomicroscope and fundus examination with indirect

Conjunctival Autofluorescence in Ocular

Surface Squamous Neoplasia ophthalmoscope were performed. Characteristics of the mass lesion including its laterality, morphology, location, extent, size, and any keratinization or pigmentation were documented. Impression cytology specimens from the OSSN lesions were obtained from all patients using standard technique. The tumour was graded according to the American Joint Commission on Cancer (AJCC)TNM classification of conjunctival carcinoma (8th edition of the AJCC Cancer Staging Manual) [16].

CUVAF images were captured using the custom-made camera system which consisted of a height-adjustable table equipped with subject headrest, camera positioning assembly, digital single-lens reflex camera, macro lens, and filtered electronic flash. Coloured low-voltage lights emitting diodes were positioned on stands in the visual field of the subject at $35^{\circ}$ to the camera-subject axis to aid fixation. All standard photographs were taken using a Nikon D100 (Nikon, Melville, NY, USA) digital camera and a 105-mm f/3.3 Micro Nikkor (Nikon) lens at fixed setting. The ultraviolet-induced fluorescence photography was based on standard principles, using a specially adapted electronic flash system fitted with ultraviolet transmission filters (transmittance range 300-400 nm, peak 365 $\mathrm{nm}$ ) as the excitation source. Subject fluorescence was recorded with a Nikon D100 (Nikon) digital camera and 105-mm f/2.8 Micro Nikon (Nikon) lens fitted with infrared and ultraviolet barrier filters. Thus, only fluorescence was recorded by the camera. Three consecutive pictures of the area of the lesion were captured per person and all demonstrated consistent patterns of autofluorescence. The image with greatest clarity was selected for further assessment. Images were saved in RGB format at the D100 settings of JPEG (1:4 compression) and high resolution. Some unwanted red light allowed by the ultraviolet transmission filter was eliminated by removal of the red channel in Adobe Photoshop (Adobe Systems Inc., San Jose, CA, USA), equivalent to the use of a cyan filter on the camera lens. Each photograph could be verified immediately after it was taken and recaptured, if necessary, to obtain an enhanced image.

CUVAF imaging was performed in all 17 eyes with OSSN and the contralateral clinically normal eyes of the study patients. Control images of pterygium and pinguecula were also captured for reference. The pattern of autofluorescence was determined by 2 experienced graders masked by clinical examination. The area of mass lesion in $\mathrm{mm}^{2}$ on standard photograph and area of autofluorescence in $\mathrm{mm}^{2}$ on ultraviolet autofluorescence photographs was calculated using ImageJ software. In lesions where multiple discrete areas of autofluorescence were present, each area was calculated separately, and the total area was represented as a summation of these. The compiled data on Microsoft Excel spreadsheet (Microsoft, Redmond, WA, USA) was analysed using Statistical Package for the Social Sciences (SPSS) software (version 20; IBM Corp., Armonk, NY). Continuous variables were assessed and summarized using mean (SD). Categorical variables were assessed using the $\chi^{2}$ test. Non-normal continuous variables were assessed using the Wilcoxon-Mann-Whitney rank-sum test.

\section{Results}

A total of 18 eyes of 17 patients were recruited during January 2017-December 2018. There were 15 male and 2 female patients, with a mean age of 57.9 years (range 10 
Fig. 1. Conjunctival autofluorescence in a patient with OSSN in the presence of negative diagnostic impression cytology. a Clinical picture of the right eye of a 55-year-old male with features of OSSN demonstrating mixed morphology with both papillary (yellow arrow) and leucoplakic components (green arrow). b Diagnostic impression cytology of the mass showed benign epithelial cells with no evidence of dysplasia (black arrows). c Conjunctival autofluorescence is present over the leucoplakic part of the tumour mass (white arrow), which helps in clinching the diagnosis in cases of confusion. $\mathbf{d}$ Post-surgical excision of the mass; dysplastic cells were seen on histopathology (blue arrow), confirming the diagnosis of OSSN.

Fig. 2. Conjunctival ultraviolet autofluorescence over the mass lesion in gelatinous OSSN. a Clinical picture of the right eye of an 85-year-old gentleman showing gelatinous type of OSSN. b Patchy areas of conjunctival autofluorescence can be seen over the tumour mass with dilated and tortuous episcleral vessels.
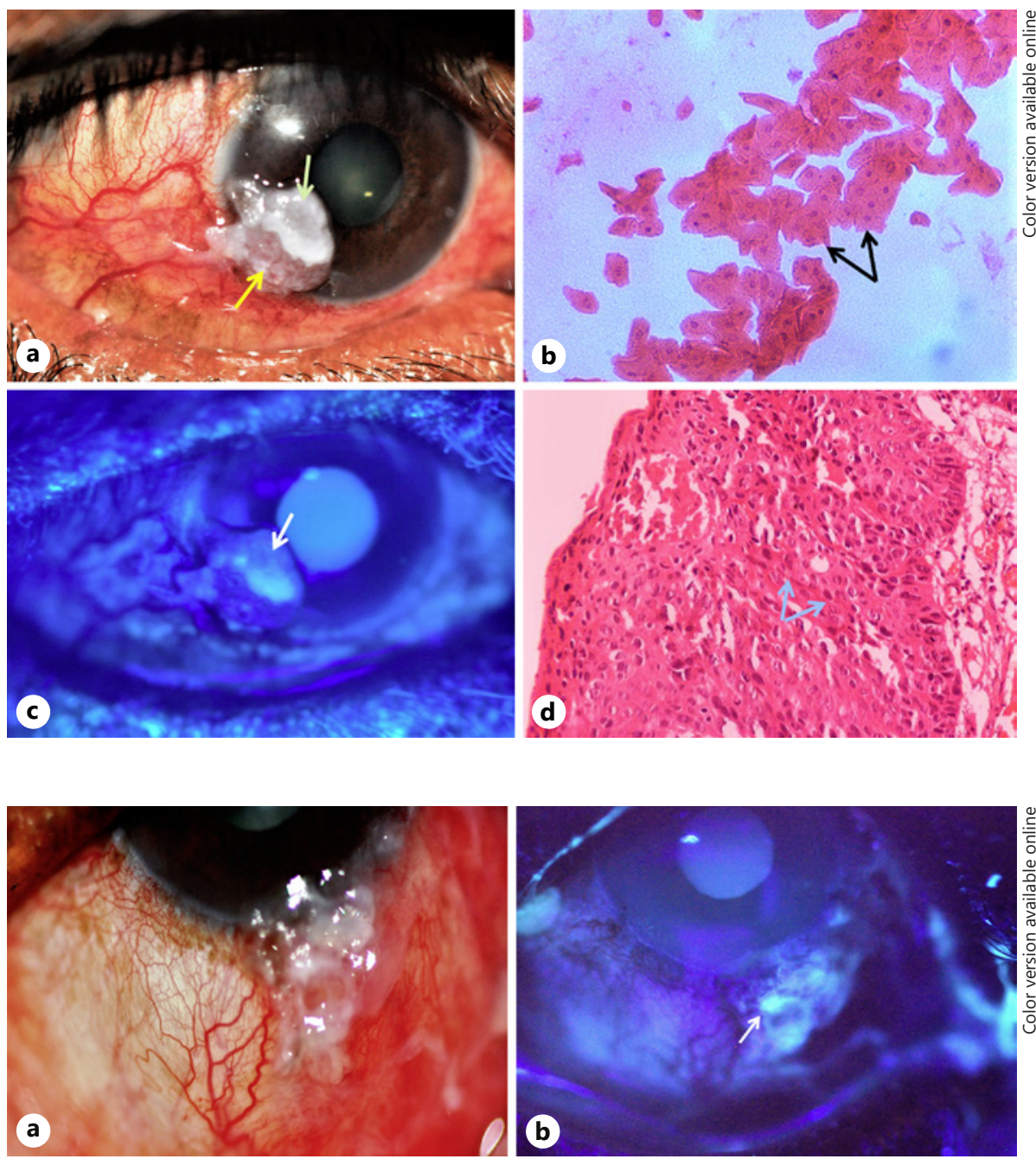

85 years). Primary OSSN was seen in 15 (88\%) patients, and $2(12 \%)$ had recurrent disease. One patient (5.88\%) had bilateral involvement. Mean logMAR visual acuity of patients was 0.527 (SD 0.38). The most common location of OSSN was temporal $(n=10 ; 55.5 \%)$ followed by nasal ( $n=6 ; 33.3 \%)$, with diffuse involvement being the least common $(n=2 ; 11.1 \%)$. Morphologically, there were 4 (22.2\%) nodular, 4 (22.2\%) leucoplakic (Fig. 1), 3 (16.7\%) gelatinous (Fig. 2), and 1 (5.5\%) each of papillary (Fig. 3), nodulo-ulcerative, and diffuse type of OSSN. Mixed morphology was present in 4 eyes $(22.2 \%)$ with 2 gelatinousleucoplakic, 1 leucoplakic-papillary, and 1 papillary-nodular type of lesion (Table 1). All tumours were either T1N0M0 or T2N0M0 as per the AJCC staging criteria. Surgical excision was performed in 7 of 18 eyes (38.9\%), and histopathological confirmation of the diagnosis of OSSN was available for these patients (Table 1). The re- maining 11 eyes $(60.1 \%)$ demonstrated tumour regression on medical treatment with either perilesional or topical interferon $a-2 b$.

Autofluorescence was displayed by 16 of 18 eyes (88.9\%) with OSSN on CUVAF imaging. The pattern of autofluorescence and clinical picture displayed in eyes with OSSN (Fig. 3a, b) was different when compared with contralateral eyes (Fig. 3c) of patients with OSSN. The OSSN lesion with prominent feeder vessels and elevated morphology was clearly seen in the CUVAF image while in the contralateral eye, a mild amount of autofluorescence was seen due to ultraviolet exposure. In eyes with pinguecula (Fig. 4a), mild autofluorescence was seen over the lesion while in cases with pterygium (Fig. 4b), the fleshy growth was interspersed with areas of autofluorescence. The overall mean area of conjunctival autofluorescence in 17 eyes with OSSN was $15.815 \mathrm{~mm}^{2}$ (10.767- 

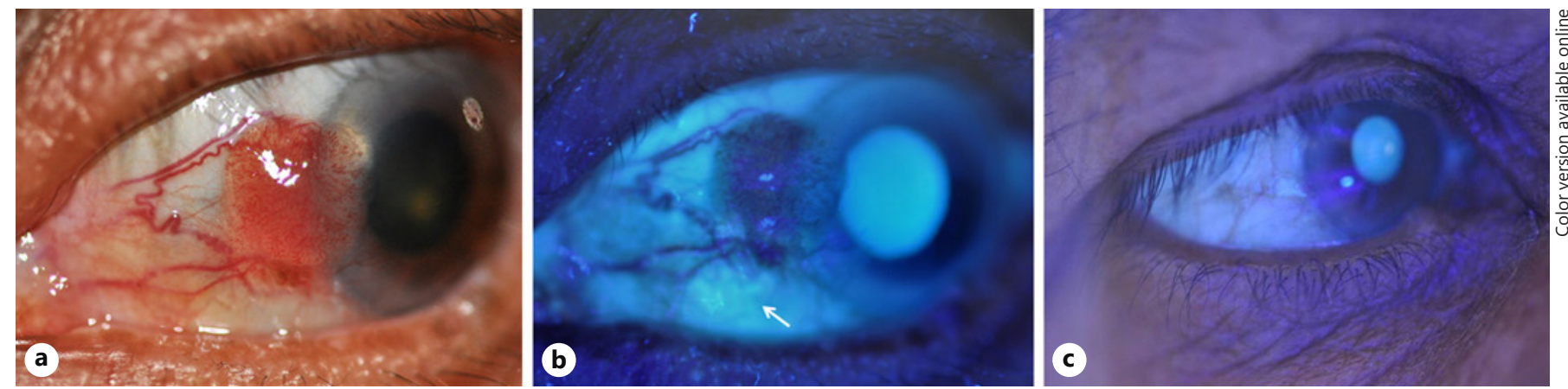

Fig. 3. Conjunctival autofluorescence is evident in the surrounding areas of the tumour. a Clinical photograph of papillary OSSN. b Conjunctival autofluorescence is seen adjacent to the tumour mass (white arrow) on ultraviolet photography. c Conjunctival ultraviolet autofluorescence imaging of the contralateral eye with no evident ocular pathology demonstrating a mild amount of conjunctival autofluorescence due to ocular ultraviolet exposure.

Fig. 4. Reference images of conjunctival ultraviolet autofluorescence in patients with pterygium and pinguecula. a Mild autofluorescence was seen over the region of the pinguecula. $\mathbf{b}$ In the case of pterygium, the fleshy growth was interspersed with areas of autofluorescence.

Fig. 5. Absent conjunctival autofluorescence in a case of pigmented OSSN. a Gelatinous OSSN with overlying pigmentary changes (green arrow). b No area of conjunctival autofluorescence is seen either over the lesion or in the surrounding area.

Fig. 6. Case of recurrent OSSN. a Clinical photograph of a previously excised OSSN with suspected recurrence (green arrow). b Autofluorescence can be seen in the suspected area of recurrence (white arrow), corroborating the clinical suspicion, which was not picked up on impression cytology.
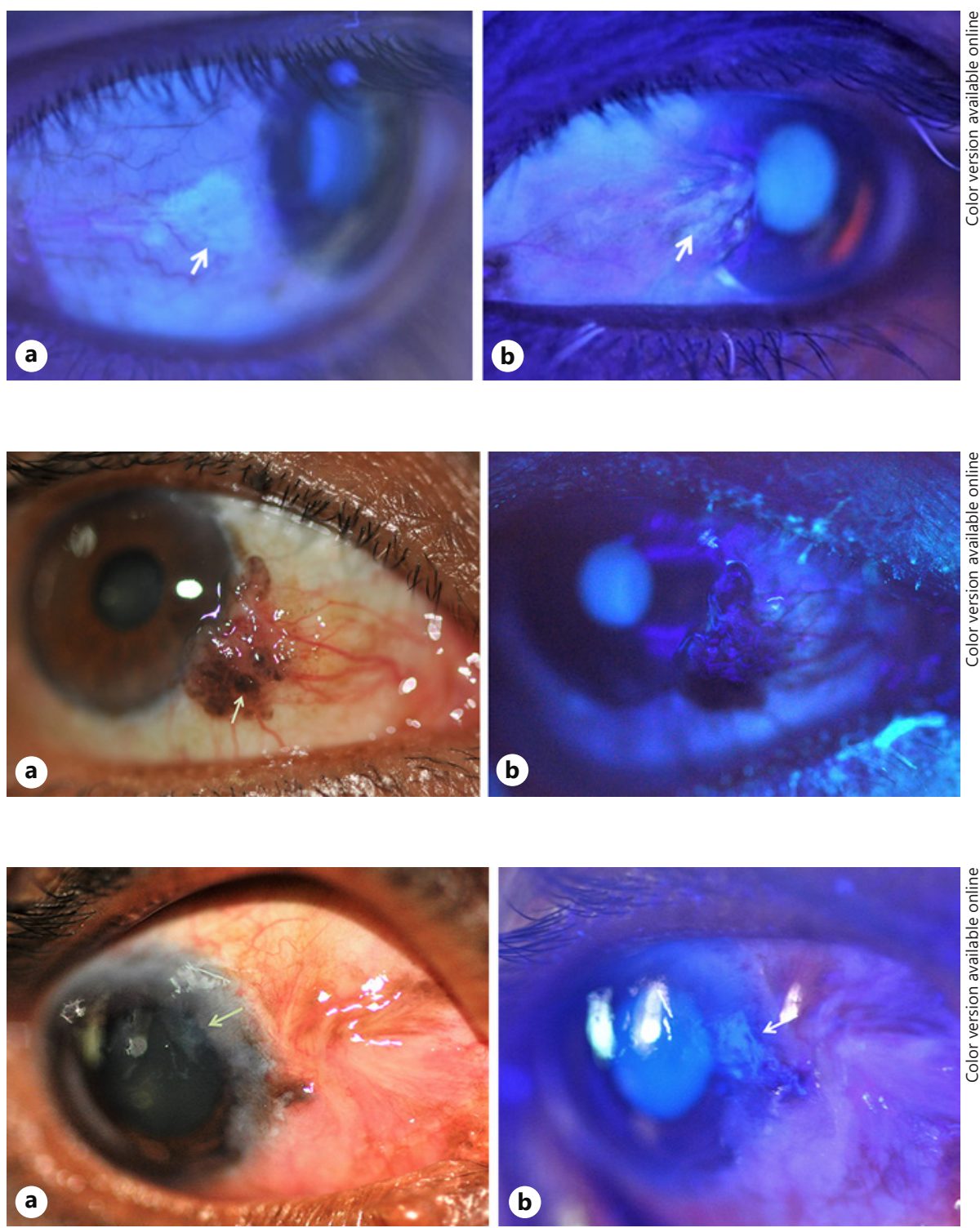


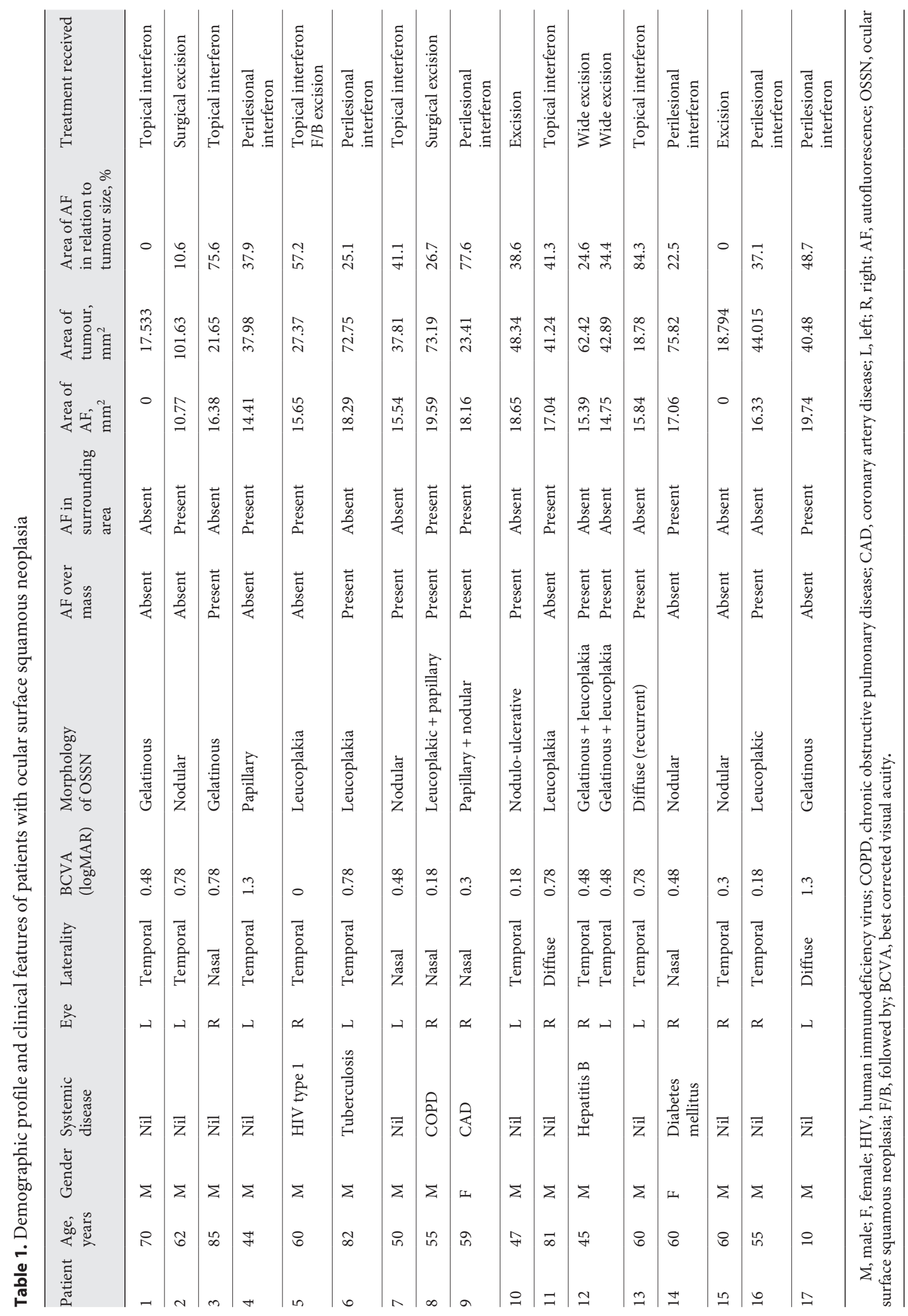




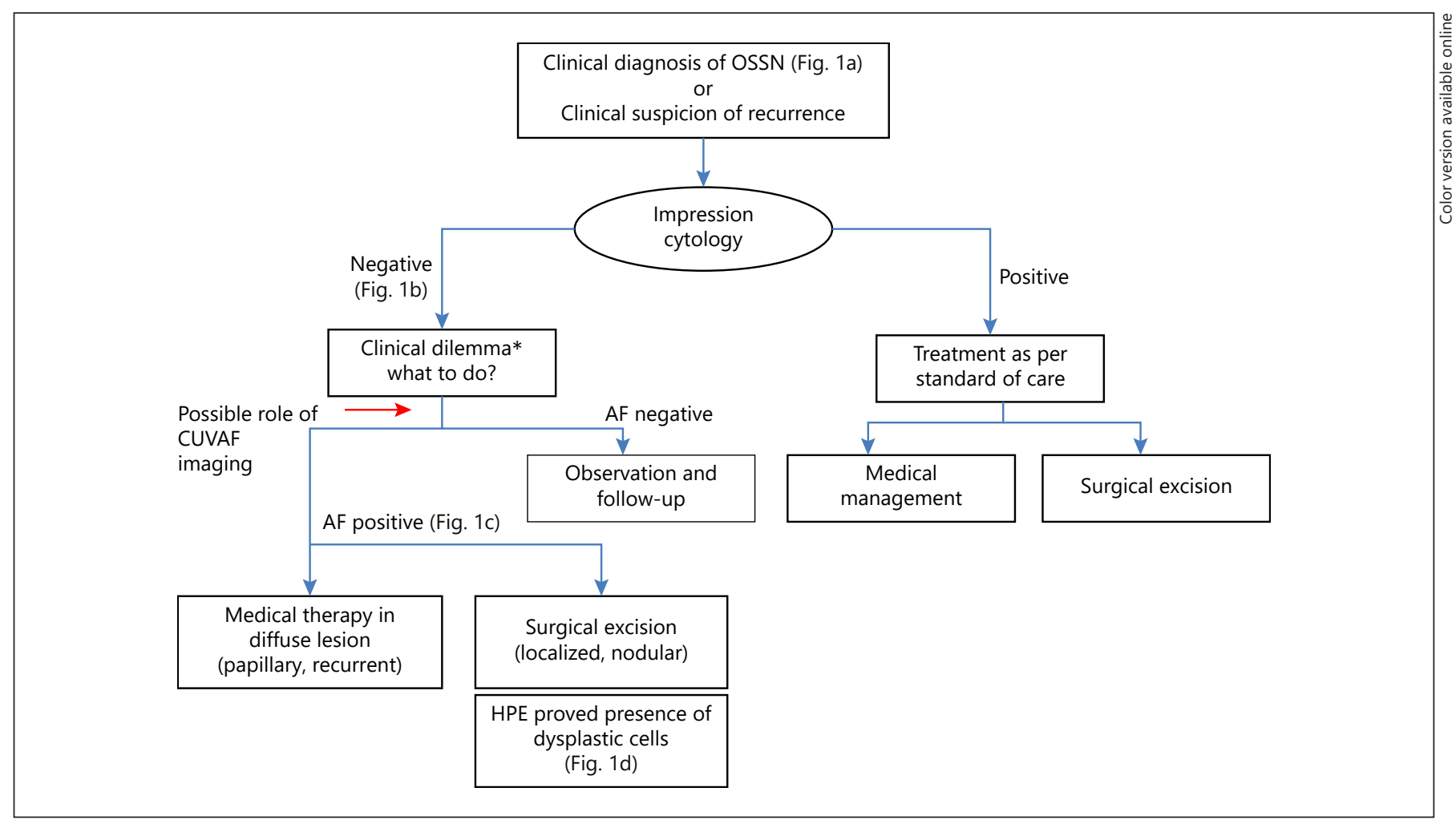

Fig. 7. Flowchart depicting the standard of care approach for the management of OSSN and the additional benefit provided with use of conjunctival autofluorescence imaging. This modality proved useful in an event of diagnostic confusion when the clinical impression did not corroborate with the results of impression cytology, as was seen in case number 8 of our study. Diagnostic dilemma, in this case a high clinical suspicion of OSSN, was solved

$19.589 \mathrm{~mm}^{2}$ ) and the median percentage area of autofluorescence observed in relation to the entire tumour was $38.3 \%$ (range 10.6-84.3\%). The most common pattern of CUVAF was patchy areas of autofluorescence over the tumour mass seen in 10 (55.5\%) eyes. In 7 eyes (38.8\%), autofluorescence was present beyond the area of visible mass lesion. None of the eyes showed a diffuse autofluorescence over the whole tumour mass. Of the 2 eyes that did not show any CUVAF, one was nodular and the other gelatinous; both were pigmented tumours (Fig. 5). In 1 eye with recurrent OSSN, autofluorescence was seen to be present in the area of clinically suspected recurrence (Fig. 6). In 8 eyes (44.4\%), CVUAF images revealed autofluorescence over the mass lesion in the presence of benign epithelial cells on impression cytology (Fig. 1c, d). All eyes with gelatinous and papillary morphology demonstrated evidence of autofluorescence with the exception of pigmented tumours. Nodular lesions did not show with the appearance of positive autofluorescent areas on conjunctival ultraviolet autofluorescence imaging. OSSN, ocular surface squamous neoplasia. CUVAF, conjunctival ultraviolet autofluorescence; AF, autofluorescence; HPE, histopathological examination. * In the absence of CUVAF imaging, therapeutic trial is the only option.

autofluorescence over the mass lesion, except in the mixed morphology type which was nodulo-ulcerative in nature (Table 1). The area of autofluorescence was significantly associated with severity of OSSN in terms of the size of the lesion $(p=0.022)$. There was no significant association of the degree of autofluorescence with age, gender, visual acuity, laterality, or presence of systemic disease in patients with OSSN.

\section{Discussion}

In recent years, there has been an increasing interest to study the association of ocular diseases with ambient solar radiation. World Health Organization has identified at least 9 diseases associated with ultraviolet radiation exposure out of which 3 are specific to the eye namely pterygium, cortical cataract and OSSN [17]. In the current 
study, we found patchy areas of conjunctival autofluorescence on ultraviolet photography in majority $(88.9 \%)$ of the eyes with OSSN.

The association of sun exposure to squamous cell dysplasia has been demonstrated in case-control and noncomparative studies [4,6]. Tulvatana et al. [18] even provided the pathological proof of sun-related damage in cases of conjunctival squamous neoplasia by demonstrating solar elastosis in the substantia propria of the conjunctival tissue. In the current study, we imaged the autofluorescence associated with lesions of OSSN using a custom-built camera system, thus providing a quick, ancillary tool for supplementing the clinical diagnosis of OSSN in the clinic itself. All cases revealed patchy areas of autofluorescence within the lesion, excepted pigmented lesions. Although no definite association of the pattern of autofluorescence with type of OSSN was found, nonpigmented papillary and gelatinous lesions demonstrated autofluorescence in all cases while none of the cases with nodular OSSN demonstrated autofluorescence over the lesion. Several studies have demonstrated that autofluorescence in the conjunctiva occurs only in some localized areas of the bulbar conjunctiva which corresponds to areas of active cellular changes due to ultraviolet and environmental exposure [19].

Surgical excision of the mass lesion with cryotherapy is considered the definitive treatment modality in selected cases such as localized and nodular varieties of OSSN. As surgical excision may not be feasible in recurrent and diffuse lesions, non-invasive diagnosis of dysplastic changes becomes crucial. Currently, impression cytology and ultrahigh-resolution anterior segment optical coherence tomography are the only non-invasive diagnostic modalities being used [20-22]. Imaging modalities usually do not provide a very sharp distinction between OSSN and benign lesions, which makes decision-making in clinical practice challenging [23]. Impression cytology harvests only superficial ocular surface epithelial cells and has a positivity rate of $78-87 \%$ in OSSN [24]. Negative reports on impression cytology can be misguiding to clinicians. Even in our study, $44 \%$ of lesions reported benign cell morphology on impression cytology but displayed autofluorescence on CUVAF imaging and dysplastic changes on histopathological examination of excised tumour tissue. Positive CUVAF may thus prove useful in challenging clinical situations where there is, for example, difficulty in diagnosis, starting therapy, and monitoring response to therapy (Fig. 7), as was seen in 8 of our cases. Obtaining targeted impression cytology specimens from the areas displaying autofluorescence can be explored in further studies.
A recent landmark in an in vitro study by Habibalahi et al. [25] reported use of multispectral autofluorescence imaging with artificial intelligence for histopathological diagnosis of OSSN. They identified neoplastic areas and boundaries between normal and neoplastic tissue in formalin-fixed OSSN specimens using false colour maps and correlated it with histopathological findings. Our study demonstrates, for the first time, in vivo conjunctival autofluorescence in patients with OSSN which corroborated with histopathological assessment of the excised tumours. Our imaging technique is a step forward towards filling up the lacunae proposed by this study and helps in achieving a clinic-based, non-contact, quick procedure for supplementing clinical diagnosis and for follow-up. It was interesting to note that autofluorescence was present in both keratinized and non-keratinized areas of OSSN and that some keratinized areas did not show any autofluorescence. Future studies for keratin markers in the excised specimens can be helpful in establishing any role of keratin in conjunctival autofluorescence.

CUVAF has been developed as an objective biomarker for demonstrating ocular ultraviolet radiation exposure [11]. Being a non-invasive, patient friendly, and resource friendly tool which does not need laboratory back-up, its future is optimistic. This gives it the additional advantage of being utilized at peripheral, outreach, and underserved areas of developing countries of tropical and sub-tropical regions, where the prevalence of OSSN is high.

Our preliminary study demonstrates the presence of autofluorescence areas in eyes with OSSN with certain limitations. It is to be noted that this technique can only assist the clinician in cases of doubt, and the results of autofluorescence imaging should not be interpreted in isolation. Further long-term prospective studies evaluating the behaviour of conjunctival autofluorescence in serial follow-up visits could be more revealing. Our study is an attempt to demonstrate the application of CUVAF imaging in supplementing the clinical diagnosis of OSSN.

\section{Statement of Ethics}

The research complies with the guidelines for human studies and was conducted ethically in accordance with the World Medical Association Declaration of Helsinki. The study was commenced after approval of the Institutional Ethics Committee (IEC-538/02.12.2016, RP-26/2016), All India Institute of Medical Sciences, New Delhi, India. Subjects (or their parents or guardians) gave their written informed consent for participation in the study. 


\section{Conflict of Interest Statement}

The authors have no conflicts of interest to declare.

\section{Funding Sources}

There are no funding sources to declare.

\section{Author Contributions}

S.Y. participated in data collection, data analysis, literature research, and writing of the paper. N.G. participated in the study design, data collection, data analysis, literature research, and writing and critical revision of the paper. R.S. and M.P. participated in data collection and data analysis. R.M. participated in the study design, data collection, and preparation of the final version of the manuscript. M.V. participated in critical revision and preparation of the final version of the manuscript. S.K. participated in data collection and preparation of the final version of the manuscript. R.T. participated in the study design, data collection, critical revision, and preparation of the final version of the manuscript.

\section{References}

1 Yang J, Foster CS. Squamous cell carcinoma of the conjunctiva. Int Ophthalmol Clin. 1997;37(4):73-85.

2 Lee GA, Hirst LW. Ocular surface squamous neoplasia. Surv Ophthalmol. 1995 May-Jun; 39(6):429-50.

3 Newton R, Ferlay J, Reeves G, Beral V, Parkin DM. Effect of ambient solar ultraviolet radiation on incidence of squamous-cell carcinoma of the eye. Lancet. 1996 May;347(9013): 1450-1.

4 Sun EC, Fears TR, Goedert JJ. Epidemiology of squamous cell conjunctival cancer. Cancer Epidemiol Biomarkers Prev. 1997 Feb;6(2): 73-7.

5 Gupta N, Sachdev R, Tandon R. Ocular surface squamous neoplasia in xeroderma pigmentosum: clinical spectrum and outcome. Graefes Arch Clin Exp Ophthalmol. 2011 Aug;249(8):1217-21.

6 Lee GA, Williams G, Hirst LW, Green AC. Risk factors in the development of ocular surface epithelial dysplasia. Ophthalmology. 1994 Feb;101(2):360-4.

7 Kamal S, Kaliki S, Mishra DK, Batra J, Naik MN. Ocular surface squamous neoplasia in 200 patients: a case-control study of immunosuppression resulting from human immunodeficiency virus versus immunocompetency. Ophthalmology. 2015 Aug;122(8):1688-94.

8 Nakamura Y, Mashima Y, Kameyama K, Mukai M, Oguchi Y. Detection of human papillomavirus infection in squamous tumours of the conjunctiva and lacrimal sac by immunohistochemistry, in situ hybridisation, and polymerase chain reaction. Br J Ophthalmol. 1997 Apr;81(4):308-13.

9 Newton R, Ziegler J, Ateenyi-Agaba C, Bousarghin L, Casabonne D, Beral V, et al.; Uganda Kaposi's Sarcoma Study Group. The epidemiology of conjunctival squamous cell carci- noma in Uganda. Br J Cancer. 2002 Jul;87(3): 301-8.

10 Monici M. Cell and tissue autofluorescence research and diagnostic applications. Biotechnol Annu Rev. 2005;11:227-56.

11 Sherwin JC, McKnight CM, Hewitt AW, Griffiths LR, Coroneo MT, Mackey DA. Reliability and validity of conjunctival ultraviolet autofluorescence measurement. $\mathrm{Br} \mathrm{J}$ Ophthalmol. 2012 Jun;96(6):801-5.

12 Sherwin JC, Hewitt AW, Kearns LS, Griffiths LR, Mackey DA, Coroneo MT. The association between pterygium and conjunctival ultraviolet autofluorescence: the Norfolk Island Eye Study. Acta Ophthalmol. 2013 Jun;91(4): 363-70.

13 Utine CA, Tatlipinar S, Altunsoy M, Oral D, Basar D, Alimgil LM. Autofluorescence imaging of pingueculae. Br J Ophthalmol. 2009 Mar;93(3):396-9.

14 Sherwin JC, Reacher MH, Keogh RH, Khawaja AP, Mackey DA, Foster PJ. The association between time spent outdoors and myopia in children and adolescents: a systematic review and meta-analysis. Ophthalmology. 2012 Oct;119(10):2141-51.

15 Ooi JL, Sharma NS, Papalkar D, Sharma S, Oakey M, Dawes P, et al. Ultraviolet fluorescence photography to detect early sun damage in the eyes of school-aged children. Am J Ophthalmol. 2006 Feb;141(2):294-8.

16 AJCC Ophthalmic Oncology Task Force. Carcinoma of the conjunctiva. In: Edge SE, Byrd DR, Carducci MA, Compton CA, editors. AJCC Cancer Staging Manual. 7th ed. New York (NY): Springer; 2009. pp. 531-3.

17 Lucas RJ, McMichael T, Smith W, Armstrong B. Solar ultraviolet radiation: global burden of disease from solar ultraviolet radiation. In: Prüss-Üstün A, Zeeb H, Mathers C, Repacholi $\mathrm{M}$, eds. Environmental Burden of Disease Se- ries, No. 13. Geneva: World Health Organization; 2006 [cited 2020 Jan 21]. Available from: http://www.who.int/entity/uv/health/solaruvradfull 180706.pdf?ua=1.

18 Tulvatana W, Bhattarakosol P, Sansopha L, Sipiyarak W, Kowitdamrong E, Paisuntornsug $\mathrm{T}$, et al. Risk factors for conjunctival squamous cell neoplasia: a matched case-control study. $\mathrm{Br}$ J Ophthalmol. 2003 Apr;87(4):396-8.

19 Wolffsohn JS, Drew T, Sulley A. Conjunctival UV autofluorescence-prevalence and risk factors. Cont Lens Anterior Eye. 2014 Dec; 37(6):427-30.

20 Paridaens AD, McCartney AC, Curling OM, Lyons CJ, Hungerford JL. Impression cytology of conjunctival melanosis and melanoma. Br J Ophthalmol. 1992 Apr;76(4):198-201.

21 Kieval JZ, Karp CL, Abou Shousha M, Galor A, Hoffman RA, Dubovy SR, et al. Ultra-high resolution optical coherence tomography for differentiation of ocular surface squamous neoplasia and pterygia. Ophthalmology. 2012 Mar;119(3):481-6.

22 Shousha MA, Karp CL, Perez VL, Hoffmann $\mathrm{R}$, Ventura R, Chang V, et al. Diagnosis and management of conjunctival and corneal intraepithelial neoplasia using ultra high-resolution optical coherence tomography. Ophthalmology. 2011 Aug;118(8):1531-7.

23 Ong SS, Vora GK, Gupta PK. Anterior Segment Imaging in Ocular Surface Squamous Neoplasia. J Ophthalmol. 2016;2016:5435092.

24 Nolan GR, Hirst LW, Bancroft BJ. The cytomorphology of ocular surface squamous neoplasia by using impression cytology. Cancer. $2001 \mathrm{Feb} ; 93(1): 60-7$

25 Habibalahi A, Bala C, Allende A, Anwer AG, Goldys EM. Novel automated non invasive detection of ocular surface squamous neoplasia using multispectral autofluorescence imaging. Ocul Surf. 2019 Jul;17(3):540-50. 